\section{BIRAND: A BASIC program to generate and test random binary sequences}

\section{DONALD J. POLZELLA and SAMUEL M. BOWER University of Dayton, Dayton, Ohio 45469}

Random binary sequences can be derived from published tables of random digits, or they may be generated by computer. For example, Fragaszy and Fragaszy's (1978) FORTRAN IV program generates "Gellermann" sequences, which are frequently used to schedule reinforcement in binary learning experiments. Gellermann sequences must meet certain criteria, including an equal number of each digit and a limited number of runs and alternations. Thus, they are actually subsets of all possible random sequences.

BIRAND is a program that generates pseudorandom sequences of binary digits that are not subject to such restrictions. Since each sequence is variously analyzed and tested for randomness, it may be used or rejected at the investigator's discretion.

BIRAND is written in BASIC and implemented on the University of Dayton Univac 70/7 computing system. To execute the program, the user merely enters the desired sequence length. BIRAND is presently dimensioned to generate a maximum of 500 binary digits, but it can be easily modified to generate considerably longer sequences. The digits ( $0 \mathrm{~s}$ and $1 \mathrm{~s})$ are generated with equal probability by the intrinsic RND function of BASIC. The function is executed initially to supply a seed value, which is used to prime the required number of calls.

Good's generalized serial test (Good, 1953; Good \& Gover, 1967) is used to test the sequence for randomness. This test compares the observed and expected frequencies of all v-plets (i.e., strings of Length $v$, where, in this case, $v=1,2,3,4$ ) composing the sequence and uses chi-square statistics to test the null hypothesis that the distinct v-plets occur equally often. The observed frequencies of 1-plets, 2-plets, 3-plets, and 4-plets are computed, as are the test statistics.

The author thanks Dick Greatorex, AFHRL/OT, Williams Air Force Base, Arizona, for his helpful comments. Requests for reprints and documented program listing should be sent to Donald J. Polzella, Department of Psychology, University of Dayton, 300 College Park Avenue, Dayton, Ohio 45469.
The generalized serial test is computed as follows:

$$
\Psi_{\mathrm{v}}^{2}=\frac{\mathrm{t}^{\mathrm{v}}}{\mathrm{N}-\mathrm{v}+1} \Sigma\left[\mathrm{n}_{\mathrm{i}}-(\mathrm{N}-\mathrm{v}+1) \mathrm{t}^{-\mathrm{v}}\right]^{2},
$$

where $t$ is the number of distinct digits, $v$ is the length of the string, $\mathrm{N}$ is the length of the generated sequence, and $n_{i}$ is the observed frequency of the $t^{v}$ distinct $v$-plets. Values of $\Psi_{\mathrm{v}}^{2}$ are only approximately chi-square distributed. The second differences on the $\Psi_{\mathrm{v}}^{2}$ values are used as test statistics. Thus, $\nabla^{2} \Psi_{\mathrm{v}}^{2}=\Psi_{\mathrm{v}}^{2}-2 \Psi_{\mathrm{v}-1}^{2}+$ $\Psi_{v-2}^{2}\left(v=1,2,3, \ldots, \Psi_{0}^{2}, \Psi_{-1}^{2}=0\right)$. These statistics are exactly chi-square distributed and are asymptotically independent, with $\mathrm{df}=\mathrm{t}^{\mathrm{v}}-2 \mathrm{t}^{\mathrm{v}-1}+\mathrm{t}^{\mathrm{v}-2}(\mathrm{v} \geqslant 2)$.

Output, which appears at the console, consists of the printed sequence, the frequency of occurrence of the distinct v-plets, and separate chi-square statistics for each value of $v$. The program will run without modification on many interactive systems. However, minor changes may be necessary. For example, if the user desires hard-copy output when implementing the program on microcomputer systems, he/she will need to amend the PRINT statements by specifying the appropriate output device number. Other possible changes relate to valid RND function calls.

It is worth noting that the portion of the program that tests the generated sequence for randomness may be called as a subroutine and used to test any stored array of $0 \mathrm{~s}$ and $1 \mathrm{~s}$. This permits the testing of sequences generated by other devices, including those generated by human subjects (Wagenaar, 1972).

\section{REFERENCES}

Fragaszy, R. J., \& Fragaszy, D. M. A program to generate Gellermann (pseudorandom) series of binary states. Behavior Research Methods \& Instrumentation, 1978, 10, 83-88.

Good, I. J. The serial test for sampling numbers and other tests for randomness. Proceedings of the Cambridge Philosophical Society, 1953, 49, 276-284.

Good, I. J., \& Gover, T. N. The generalized serial test and the binary expansion of $\sqrt{2}$. Journal of the Royal Statistical Society (Series A), 1967, 130, 102-107.

WAGENAAR, W. A. Generation of random sequences by human subjects: A critical survey of literature. Psychological Bulletin, $1972,77,65-72$.

(Accepted for publication February 5, 1982.) 